\title{
RICE SHAPE PARAMETER DETECTION BASED ON IMAGE PROCESSING
}

\author{
Hua Gao ${ }^{1, *}$, Yaqin Wang ${ }^{1,2}$, Pingju Ge ${ }^{1}$ \\ ${ }^{1}$ College of Information Science \& Engineering, Shandong Agricultural University, Taian, \\ China, 271018 \\ ${ }^{2}$ College of Geo Info. Science and Technology, Shandong University of Science and \\ Technology, Tsingdao, China, 266510 \\ * Corresponding author, Address: College of Information Science \& Engineering, Shandong \\ Agricultural University, Taian, 271018, P. R. China, Tel: +86-538-8242497, Fax: + 86-538- \\ 8249275,Email: gaoh@sdau.edu.com
}

Abstract: Based on image processing technology, the detection, classification and feature extraction for plant grain shape are performed in this paper. Taking rice grain as an example, the shape detection and description method of similar round object are studied firstly. Then a grain shape description method based on 8 feature points of rice grain boundary is proposed. Aiming at rice seed detection, a simple image size calibration method based on black-white grid is put forward too. Finally, an extraction algorithm for 8 feature points is presented.

Keywords: rice grain, shape description, feature extraction, image processing

\section{INTRODUCTION}

The grain shape of plant seed is one of the important parameters for seed quality detection and classification (Ren et al., 2004). It is a hot issue all over the world to detect and classify plant grain shape based on image processing technology. At the same time, it has long been recognized as a difficult problem. Parameters describing plant grain shape are determined by grain shape feature and research objective. For some researches, only one point or several points shape features of object are needed to describe the shape (Van Eck et al., 1998). While for some other researches, the whole shape features 
of object are described in order to distinguish this kind of object from others or to find shape parameters related with its own special features (Heinemann et al., 1994; Wang et al., 1995). Here some parameters such as area, eccentricity, tightness, percentage of elongation, inertia center, ratio of grain length to width, ratio of grain width to thickness, grain sphericity, grain roundness and Fourier descriptor, are used to describe the object. For example, Ma xiaoyu selects four wheat shape correction coefficients to describe variety difference (Ma et al., 1999). Zhang cong proposes an ellipse matching-based object (rice grain) azimuth location and boundary description method (Zhang et al., 2006). Huang xingyi et al. use area, perimeter, roundness degree and rectangle degree to describe rice shape (Huang et al., 2003). In order to find suitable rice grain shape description method and to improve detection and classification precision, it is imperative that some key parameters should be found to describe grain feature exactly. Taking rice grain as an example, the shape detection and description method of similar round object are presented in this paper. Moreover, based on 8 feature points of rice boundary, the grain shape description method is proposed and its realization algorithm is given.

In computer visual technology-based measurement, image geometric distortion caused by camera lens may result in an inaccurate surveying result, so it is necessary to calibrate the camera before shooting object. Aiming at rice seed detection, a simple image size calibration method based on black-white grid is put forward too.

\section{RICE IMAGE ACQUISITION AND IMAGE PIXEL-SIZE CALIBRATION}

In order to decide rice image pixel size, it is necessary to calibrate camera before rice image acquisition. At present, most camera calibration algorithms calibrate internal and external parameter at the same time to obtain the relations among three coordinate systems (Huang et al., 2002; Zhong et al., 2005). But in seed detection, the most interested thing to be concerned by people is the relationship between image size and actual size of seed instead of internal or external parameter of camera. Therefore, it is no need to use traditional complicated calibrating method when the seed is detected.

In this paper, the image distortion model is simplified. It is supposed that there is only image size distortion and no image angle distortion. Based on this supposition, a simple image size calibrating method based on blackwhite grid is proposed.

The specific method is described as follows: 
1. Install and fix the camera vertically above the object desk (See figure 1).

2. Shoot black-white grid image before seed image shoot (every blackwhite grid is a one-inch square, see figure 2).

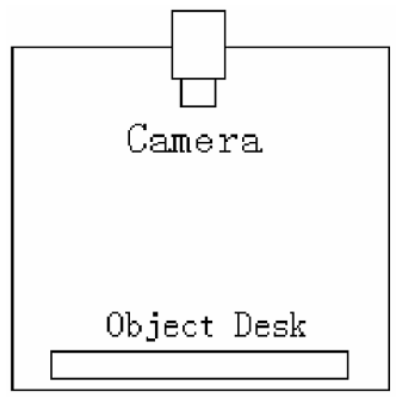

Figure 1. Sketch map of camera installation structure

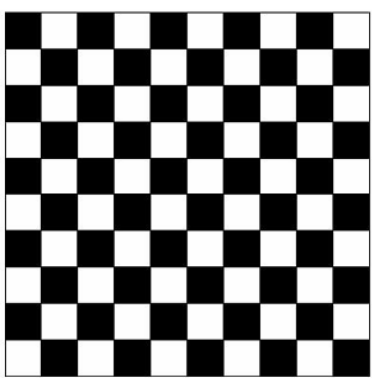

Figure 2. Black-white grid image used for size calibration

3. Search corner point coordinate of grid and get corner point coordinate matrix:

$$
G=\left|\begin{array}{cccc}
g_{0,0} & g_{0,1} & \ldots & g_{0, n-1} \\
g_{1,0}, & g_{1,1} & \ldots & g_{1, n-1} \\
\ldots & \ldots & \ldots & \ldots \\
g_{n-1,0} & g_{n-1,1} & \ldots & g_{n-1, n-1}
\end{array}\right|
$$

Here, $g_{i, j}=\left(x_{i, j}, y_{i, j}\right)$ is corner point coordinate except boundary corners. Because the boundary of black-white grid image is illegible, when calculating the corners, grid's region image is obtained firstly on the basis of region growing algorithm. Then four region inner points are searched. Finally, the cross point of four inner points are calculated. This cross point is the needed corner coordinate (See figure 3). 


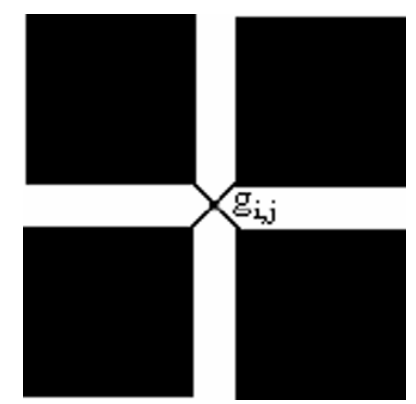

Figure 3. Sketch map of corner coordinate calculation

4. Calculate the pixel size.

The pixel size of core-point in every grid of image is:

$$
W_{i, j}=\frac{1}{x_{i, j}-x_{i+1, j}} \quad H_{i, j}=\frac{1}{y_{i, j}-x_{i, j+1}}
$$

Here $\mathrm{W}_{\mathrm{i}, \mathrm{j}}$ and $\mathrm{H}_{\mathrm{i}, \mathrm{j}}$ stand for the pixel size (unit: inch) of core-point along $\mathrm{x}$ direction and y direction separately in grid of row $i$ and column $j$.

5. Calculate every pixel's width $\mathrm{W}_{\mathrm{ij}}$ and height $\mathrm{H}_{\mathrm{ij}}$ based on liner difference, and establish the matrix:

$$
\Delta=\left|\begin{array}{cccc}
\delta_{0,0} & \delta_{0,1} & \ldots & \delta_{0, m-1} \\
\delta_{1,0}, & \delta_{1,1} & \ldots & \delta_{1, m-1} \\
\ldots & \ldots & \ldots & \ldots \\
\delta_{n-1,0} & \delta_{n-1,1} & \ldots & \delta_{m-1, m-1}
\end{array}\right|
$$

Here $\delta_{\mathrm{i}, \mathrm{j}}=\left(\mathrm{W}_{\mathrm{ij}}, \mathrm{H}_{\mathrm{ij}}\right)$ is the width and height of pixel.

With this matrix we can calculate rice image pixel size accurately. In order to simplify the algorithm, we can substitute rice grain centroid pixel's coefficient for the whole grain image pixel' coefficient when the rice grain size is calculated.

6. Shoot the rice grain: In order to establish the mapping relationship between rice image and pixel size coefficient matrix, it is imperative to put the rice grain on the center of the desk equably.

\section{DESCRIPTION OF RICE GRAIN SHAPE}

Rice grain shape is very complex and is affected by many factors. There are many methods to describe grain shape at present, but they do have some restrictions. For example, some methods, such as area method, eccentricity methods, percentage of elongation, inertia center, ratio of grain length to width, ratio of grain width to thickness, grain sphericity, and grain 
roundness, have a lower precision. Fourier descriptor and some other methods are more accurate but they need to do a large amount of calculations and have a bad visualizability. A new method is presented in this paper, which describes grain shape with 8 selected points on grain boundary. As is shown in figure 4(d). This method has several advantages when it is used to describe grain shape, such as simple calculation, small amount of data, accurate description etc. The specific algorithm is described as follows:

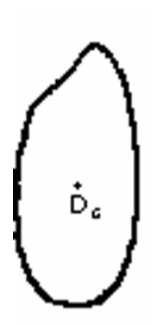

a

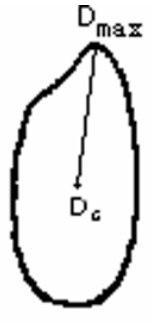

b

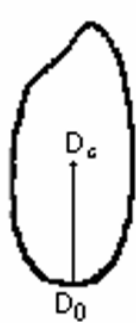

C

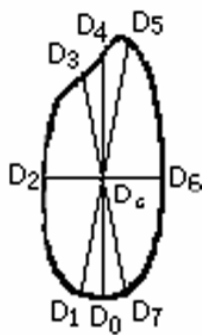

d

Figure 4. Searching of 8 feature points

1. Calculate centroid coordinate $D_{c}$

Centroid coordinate can be expressed by either average of rice boundary coordinate or region barycenter. The former one is used in this paper. See figure 4(a).

$$
x_{c}=\frac{1}{M} \sum_{i=0}^{M-1} x_{i} \quad y_{c}=\frac{1}{M} \sum_{i=0}^{M-1} y_{i}
$$

2. Find point $D_{\max }$ in the boundary which has the longest distance from centroid.

$L_{i}$ is the distance from boundary point $D_{i}$ to centroid $D_{c}$

$$
L_{i}=\sqrt{\left(x_{i}-x_{c}\right)^{2}+\left(y_{i}-y_{c}\right)^{2}}
$$

Find maximum $\mathrm{L}_{\max }$ along the boundary to get the coordinate of $\mathrm{D}_{\max }$. See figure 4(b).

3. Find the longest distance point $\mathrm{D}_{0}$ within boundary local region, which is on the opposite side of maximum distance point.

Find maximum value along boundary within the scope of $\pm \pi / 4$ opposite to $\mathrm{D}_{\max }$ to get the coordinate of $\mathrm{D}_{0}$. This point is the first one of 8 points. See figure 4(c).

4. Start from $D_{0}$ and search the boundary point clockwise to find $D_{1}$ to $D_{7}$ one by one. The interval angle between every two feature points are л/16, $7 л / 16,7 л / 16$, л/16, л/16, 7л/16, 7л/16. See figure $4(\mathrm{~d})$. The angle can be calculated according to following formula: 


$$
\theta=a c \tan \frac{\left(y_{c}-y_{f}\right)}{\left(x_{c}-x_{f}\right)}-a c \tan \frac{\left(y_{c}-y_{a}\right)}{\left(x_{c}-x_{a}\right)}
$$

\section{Image rotation}

When shooting rice image, the direction of grain is random. In order to standardize image, it is needed to rotate the image boundary. The rotation angle is the include angle between line $\mathrm{D}_{0} \mathrm{D}_{\mathrm{c}}$ and perpendicular line. Here $\mathrm{D}_{\mathrm{c}}$ is centroid point.

$$
\alpha=\operatorname{actan} \frac{\left(x_{0}-x_{c}\right)}{\left(y_{c}-y_{0}\right)}
$$
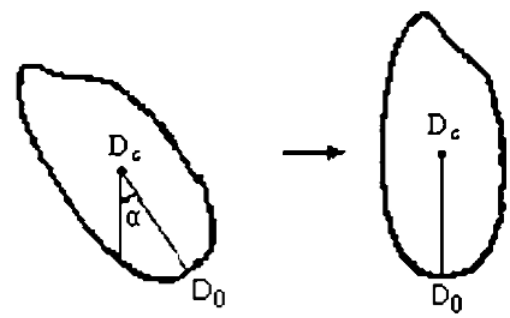

Figure 5. Image rotation

Rotation transformation is performed as follows. See figure 5.

$$
\left[\begin{array}{l}
x \\
y \\
1
\end{array}\right]=\left[\begin{array}{ccc}
\cos \alpha & -\sin \alpha & 0 \\
\sin \alpha & \cos \alpha & 0 \\
0 & 0 & 1
\end{array}\right]\left[\begin{array}{l}
x \\
y \\
1
\end{array}\right]
$$

\section{Flip horizontal}

For the same rice grain, two opposite placement may result in two different images, see figure 6 . In order to let 8 describing points have the same sequence, all the embryos are on the top-left in the image. For every nonstandard grain image, flip horizontal should be done along the perpendicular line crossing centroid point.

Supposed that $\mathrm{L}_{3}$ is the distance from point $\mathrm{D}_{3}$ to centroid point, and $\mathrm{L}_{5}$ is the distance from point $\mathrm{D}_{5}$ to centroid point. Let's compare $\mathrm{L}_{3}$ with $\mathrm{L}_{5}$, if $\mathrm{L}_{3}>\mathrm{L}_{5}$, then to flip grain image horizontal, otherwise, no flip. The flip formula is:

$$
\left[\begin{array}{l}
x \\
y \\
1
\end{array}\right]=\left[\begin{array}{ccc}
-1 & 0 & f \text { Width } \\
0 & 1 & 0 \\
0 & 0 & 1
\end{array}\right]\left[\begin{array}{l}
x \\
y \\
1
\end{array}\right]
$$




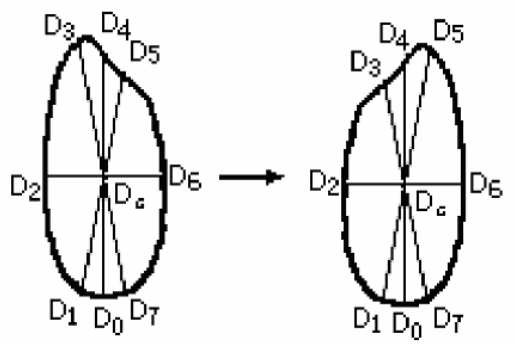

Figure 6. Flip image horizontal

\section{Measurement unit conversion}

All the measurement units above are pixel. While in practice, the measurement unit should be conversed to length unit. Therefore, the coordinate of every grain's centroid point $\mathrm{D}_{\mathrm{c}}$ is recorded, and element $\delta_{\mathrm{i}, \mathrm{j}}$ is used as grain size coefficient. Here $\delta_{\mathrm{i}, \mathrm{j}}=\left(\mathrm{W}_{\mathrm{ij}}, \mathrm{H}_{\mathrm{ij}}\right)$, is coming from pixel size coefficient matrix. Then, the measurement unit conversion will be performed.

\section{CONCLUSIONS}

Too fine description of agricultural product grain shape is very complicated. Sometimes it will make trouble for analysis and recognition. In this paper, the method to describe rice grain shape with 8 feature points is not only simple, but also high efficient. Some key feature parameters of rice are contained in the eight data, such as length, width, perimeter, area as well as ellipse approximate degree. Combined with neural network in the experiment, this method has a good effect on the recognition and classification of rice.

The simple method to calibrate rice image size proposed in this paper is also fit to every fixed distance shoot image size calibration.

\section{REFERENCES}

Heinemann P H et al. 1994, Grading of mushrooms using a machine vision system. Transactions of ASAE. 37(5):1671-1677.

Huang Fengrong, Liu Jiaomin, Sun Zhuangzhi 2002, Distance measurement system based on new method of determining the three-dimensional position and orientation of object from a single view. Computer Engineering and Applications. 38(4):236-238.

Huang Xingyi et al. 2003, Inspection of chalk degree of rice using genetic neural network. Transactions of The Chinese Society of Agricultural Engineering. 19(3):137-139. 
J W Van Eck et al. 1998, Accurate measurement of size and shape of cucumber fruits with image analysis. J Aagric Engng Res. (70):335-343.

Ma Xiaoyu, Lei Detian 1999, Study on the mechanical Rheological properties of soybean and wheat grain grown in northeast China. Transactions of The Chinese Society of Agricultural Engineering. 15(3):70-75

Ren Xianzhong, Ma Xiaoyu 2004, Research advances of agricultural product grain shape identification and current situation of its application in the engineering field. Transactions of The Chinese Society of Agricultural Engineering. 20(3):276-280.

Wang Fengyuan, Zhou Yiming 1995, Seed shape detection and measurement with computer image processing. Transaction of the Chinese society of agricultural machinery. 26(2):52-57.

Zhang Cong, Guan Shuan 2006, Rice figure identification based on an image analysis. Cereal \& Feed Industry. 6: 5-7

Zhong Zhiguang, Yi Jianqiang, Zhao Dongbin 2005. A geometric approach for camera calibration based on point pairs. Robot. 1(27):31-35 